\title{
The effect of enclosure on the allocation of visually induced movement
}

LEONARO BROSGOLE AND PATRICIA M. WHALEN

NAVAL TRAINING DEVICE CENTER

Question was raised as to whether enclosure is a factor which determines the allocation of visually perceived movement. Enclosure was found to influence induced movement by virtue of the manner in which it affects the symmetry of visual space as opposed to being a perceptual selection principle.

Karl Duncker (1929) made a major logical contribution by distinguishing between the initiation of visually perceived movement and those factors which determine its allocation. Initiation refers to that condition necessary for signaling the mere presence of movement within the field, while allocation denotes the manner and extent to which that total amount of motion is distributed among the various objects in visual space. Duncker demonstrated this distinction by subjecting the induced motion phenomenon to two characteristicall:; different sets of conditions.

In one instance, a stationary target was fixated in the presence of another similar object whlch moved back and forth subliminally. The continuous position change between the two objects was sufficient to generate an apparent motion in the stationary target, so that they were both seen to approach and withdraw from one another symmetrically.

In the second case, the stationary target was surrounded by a rectangular frame which moved back and forth laterally. Even though the relative position change generated by the surround was equivalent to that produced by the displacing object, in the above instance, the stationary target now carried the full amount of induced motion and the frame appeared to remain more or less at rest.

Duncker concluded that position change was necessary for signaling object motion in visual space. Relative displacement alone, however, did not selectively discriminate between the objects in assigning motion within the visual field. As a result, the two participants, in the former case, shared equally in the total a mount of movement. Because the target seemed to carry the full amount of motion in the second instance, the feature of enclosure was held to be a principle determining the manner in which motion is allocated.

Enclosure could not legitimately be used to explain Duncker's findings, however, because it was derived as a principle from those very same observations. It would be tautological to employ it in such a manner. In addition, since enclosure has only intuitive appeal, without any fundamental substance, it is impossible to predict precisely to what extent a target would be induced to move by a surrounding object relative to an adjacent one. The purpose of this paper, therefore, is to provide an alternative explanation of the effect of enclosure on induced movement.

Contrary to Duncker's interpretation, Brosgole (1966) demonstrated that induced motion is based upon the subjective displacement of a target in phenomenal space. He basically found that when a stationa ry object was surrounded by a rectangular frame, which moved back and forth laterally, the apparent straight ahead shifted along with the center of the frame so as to remain symmetrically located within the visible field. As a result, the target continuously displaced relative to the apparent median plane and, therefore, was seen to move.

When a target is enclosed within a frame, visual space is limited by the vertical borders of the surround with its horizontal midpoint serving as the center of visual stimulation. The situation is markedly changed by replacing the frame with an object placed adjacent to the target-let us say one which displaces back and forth in the same manner as the frame. Now the boundaries of visual space are determined by the target and this second stimulus with the center of the field residing midway between them. The apparent straight ahead, therefore, would tend to be localized between the two objects instead of being anchored about the adjacent stimulus which resides in that position previously occupied by the middle of the frame. In other words, enclosure should result in a twofold increase in the displacement of the apparent median plane and, hence, in the amount of induced motion perceived. The following experiment was designed to critically test this premise.

\section{Apparatus}

The apparatus has been described elsewhere in detail (Brosgole, 1967). Essentially, it consisted of a wooden screen (60 in. high $\times 30$ in. wide) upon which stimuli could be mounted. The screen was remotely driven back and forth horizontally, 22 in. across space, at a rate of $20^{\prime}$ of visual arc per sec. At the end of the 22-in. journey the screen activated a relay reversing its direction of travel.

Provision was made for housing a target directly in front of the screen. The $S$ was able to move the target remotely, $30 \mathrm{in}$. to the left or right of the objective median plane, by rotating a knob fixed to the shaft of a Selsyns synchronous motor. The position of the screen and target was plotted on a Brush strip chart recorder. Stimuli

The stimuli consisted of two 1 in. diameter luminous spheres and a 24 in. $x 30$ in. horizontal rectangular frame with 1 in. wide luminous borders. 


\section{Procedure}

The experiment consisted of two conditions with a separate group of 12 serving in each. It took place in total darkness with $S$ positioned in a head and chin rest $10 \mathrm{ft}$. from the apparatus. In the first condition, $\mathrm{S}$ viewed the spherical target, located in the objective median plane, which was surrounded by the frame. He was required to adjust the target to the subjective straight ahead and was then advised that $E$ might arbitrarily move the target and/or the frame in the same or opposite directions. The S's task was to ignore the frame and attempt to hold the sphere directly straight ahead (i.e., perpendicular to the midline of the body) at all times by turning the control knob appropriately. The frame was then set into motion. It displaced from center to the right, back through center to the left and back to true center again for 10 complete cycles. The S's continuous adjustments provided a record of the amount of motion the frame tended to induce in the target.

The second condition was identical to the first, except that a second luminous sphere served as the inducing object instead of the frame. Its spatial location coincided with the horizontal center of the frame. It was positioned 1 in. above the target, however, so as to prevent $S$ from confusing it with the target when the two crossed over. Subjects

Ten males and 14 females participated in this study. Ranging in age from 18-24, their mean age was 20.1 years.

\section{Results and Discussion}

The S's setting of the target was noted when the inducing stimulus was at its extreme right and left positions. The difference between these settings represented the range of motion induced in the target for a given cycle or trial. The mean range was calculated for each $\mathrm{S}$ over 10 trials. Averaging across Ss, the target was induced to move $6.87 \mathrm{in}$. by the frame compared to 3.23 in. by the adjacent stimulus. The two conditions significantly differed according to the Mann-Whitney $U$ Test $(U=31, p=.01)$. The fact, then, that enclosure had enhanced the induced effect by a factor of 2.1 substantiates our initial speculation.

There are several ancillary findings which may be of some interest. For one, it has been our general feeling that induced motion tends to decrease as a function of time of inspection. To test this notion, the first five trials were compared to the latter five in each of the two conditions. Average error ranges of 7.0 in. (first five trials) and 6.7 in. (last five trials) were found in the first condition, while 3.2 in. and 3.3 in. were obtained in the second. The data, then, seem to demonstrate that our suspicion was without foundation-at least for approximately $10 \mathrm{~min}$. of observation.

Secondly, we have repeatedly gained the impression that the fixated target does not always appear to move in a direction opposite to the inducing stimulus but, at times, seems to travel along with it. Although this was found only in certain instances in the first condition, to be specified below, it did occur during 18 trials in the second condition, or in $15 \%$ of the cases, verifying our own personal observations.

The data were analyzed in an attempt to determine whether or not motion is induced at an equal rate throughout the entire range of visual space. Changes in the location of the target were noted for every 2.75 in. of movement of the frame. The Friedman Two Way Analysis of Variance indicated that changes in the position of the frame did generate significant alterations in the rate at which the target was induced to move $\left(\mathrm{Xr}^{2}=\right.$ 60.29 , df $=15, p<.001$ ). Using the Wilcoxon Test, each position of the frame was compared to its succeeding one. The only differences found were in the two instances in which the frame reversed direction, where there was a reliably marked slowdown in the speed of induced movement (in each case $T=0, N=12, p<.01$ ). In fact, six Ss continued to see some movement in the same direction even though the frame had already reversed. ${ }^{1}$ These data negate the contention that a target is induced to move at a greater velocity when it is closest to the borders of its surround and least when it is located in the center.

Leaving these tangential issues aside and returning to the main findings, there are many factors which can conceivably affect the allocation of motion. For example, active participation may be the basis for assigning motion to the self as opposed to the environment. Enclosure, however, is clearly not such a selective principle. Its effect is rather based upon the manner in which it affects the symmetry of visual stimulation.

\section{References}

Brosgole, L. An analysis of induced motion. Port Washington: Naval Training Device Center, Tech. Rept.IH-48, February 1966. Brosgole, L. Induced autokinesis. Percept. \& Psychophys., 1967, 2, 69-73.

Duncker, K. Über induzierte Bewegung (Ein Beitrag zur Theorie Optisch Wahrgenommener). Psychol. Forsch., 1929, 12, 180-259.

\section{Note}

1. Three additional Ss had consistently seen the target change direction prior to the reversal of the frame. 